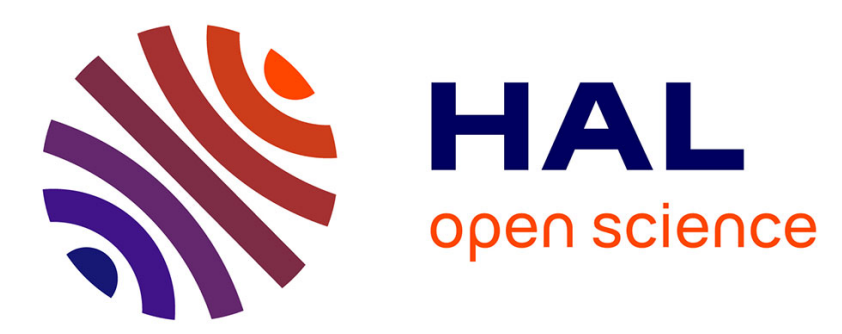

\title{
Structures sociales et structures génétiques dans les populations humaines
}

\author{
R. Chaix, G. Ly, R. Laurent, S. Lafosse, B. Toupance, C. Monidarin, O. \\ Evrard, Frédéric Bourdier, S. Pavard
}

\section{- To cite this version:}

R. Chaix, G. Ly, R. Laurent, S. Lafosse, B. Toupance, et al.. Structures sociales et structures génétiques dans les populations humaines. Bulletins et Mémoires de la Société d'anthropologie de Paris, 2020, 32 (1-2), pp.18-23. 10.3166/bmsap-2020-0071 . mnhn-02917408

\section{HAL Id: mnhn-02917408}

\section{https://hal-mnhn.archives-ouvertes.fr/mnhn-02917408}

Submitted on 15 Dec 2020

HAL is a multi-disciplinary open access archive for the deposit and dissemination of scientific research documents, whether they are published or not. The documents may come from teaching and research institutions in France or abroad, or from public or private research centers.
L'archive ouverte pluridisciplinaire HAL, est destinée au dépôt et à la diffusion de documents scientifiques de niveau recherche, publiés ou non, émanant des établissements d'enseignement et de recherche français ou étrangers, des laboratoires publics ou privés. 
archives-ouvertes

\title{
Structures sociales et structures génétiques dans les populations humaines
}

\author{
R. Chaix, G. Ly, R. Laurent, S. Lafosse, B. Toupance, C. Monidarin, O. \\ Evrard, F. Bourdier, S. Pavard
}

\section{To cite this version:}

R. Chaix, G. Ly, R. Laurent, S. Lafosse, B. Toupance, et al.. Structures sociales et structures génétiques dans les populations humaines. Bulletins et Mémoires de la Société d'anthropologie de Paris, Springer Verlag, 2020, 10.3166/bmsap-2020-0071 • mnhn-02917408

\section{HAL Id: mnhn-02917408}

\section{https://hal-mnhn.archives-ouvertes.fr/mnhn-02917408}

Submitted on 15 Dec 2020

HAL is a multi-disciplinary open access archive for the deposit and dissemination of scientific research documents, whether they are published or not. The documents may come from teaching and research institutions in France or abroad, or from public or private research centers.
L'archive ouverte pluridisciplinaire HAL, est destinée au dépôt et à la diffusion de documents scientifiques de niveau recherche, publiés ou non, émanant des établissements d'enseignement et de recherche français ou étrangers, des laboratoires publics ou privés. 


\title{
Structures sociales et structures génétiques dans les populations humaines
}

\author{
Social Structures and Genetic Structures in Human Populations
}

\author{
R. Chaix $\cdot$ G. Ly $\cdot$ R. Laurent $\cdot$ S. Lafosse $\cdot$ B. Toupance $\cdot$ C. Monidarin $\cdot$ O. Evrard $\cdot$ F. Bourdier $\cdot$ S. Pavard
}

Reçu le 3 février 2020; accepté le 18 février 2020

Résumé Dans cette note, nous revenons sur les grandes lignes de la conférence donnée lors des journées de la SAP 2019 concernant le champ actuel de l'ethnogénétique, qui s'intéresse entre autres à l'influence des systèmes de parenté sur la diversité génétique des populations humaines. Nous présentons certains des résultats principaux d'une étude ethnogénétique menée en Asie du Sud-Est dans douze populations présentant des systèmes de parenté variés, à filiation patrilinéaire, matrilinéaire et cognatique. Les données ethno-démographiques et génétiques collectées montrent que les processus de résidence, de filiation et d'alliance façonnent la diversité génétique des populations humaines. Ce champ de recherche ouvre ainsi de nouvelles perspectives : mieux connaître, à partir des données génétiques, la dynamique des systèmes de parenté actuels, et retracer leur évolution à travers le temps grâce aux données archéogénétiques. Cependant, les données ethno-démographiques montrent également que le niveau d'observance des règles varie entre populations catégorisées dans un même système de parenté, ce qui module les signatures génétiques laissées par ce système de parenté et invite à une certaine prudence concernant l'inférence des structures sociales du passé à partir des données archéogéné-

R. Chaix $(\bowtie) \cdot$ G. Ly $\cdot$ R. Laurent $\cdot$ S. Lafosse $\cdot$ B. Toupance S. Pavard

Unité Eco-anthropologie (EA), Muséum national d'Histoire naturelle, CNRS, Université de Paris, F-75016, Paris, France

e-mail : chaix@mnhn.fr

C. Monidarin

Rodolphe Merieux Laboratory and Faculty of Pharmacy

of University of Health Sciences, Phnom Penh, Cambodia

O. Evrard

Patrimoines Locaux, Environnement et Globalisation (PALOC), MNHN-IRD, 75006, Paris, France

F. Bourdier

Unité 201 Développement et Sociétés (DEVSOC), IEDES/IRD,

Panthéon Sorbonne, Paris, France. tiques. L'impact du « puzzle matrilinéaire » sur la diversité génétique autosomale est également discuté.

Mots clés Système de parenté · Organisation sociale · Résidence $\cdot$ Puzzle matrilinéaire $\cdot$ Diversité génétique

Abstract In this note, we summarize the talk given at the SAP conference 2019 regarding the field of ethnogenetics, that studies the influence of kinship systems on the genetic diversity of human populations. We will present some of the main results of an ethnogenetic study led in twelve Southeast Asian populations exhibiting various kinship systems, with matrilineal, patrilineal and cognatic descent. This study contributes to highlight that processes of residence, descent and alliance shape the genetic diversity of human populations. This opens new perspectives, notably understanding better the present dynamics of kinship systems thanks to genetic data, and tracing back their evolution through time thanks to archeogenetic data. However, this work also shows that the signatures left by a given type of social organization are variable depending on the extent to which the rule is observed, inviting to be careful when inferring past social systems from archeogenetic data. The genetic impact of the « matrilineal puzzle » is also discussed.

Keywords Kinship systems · Social organisation · Residence $\cdot$ Matrilineal puzzle $\cdot$ Genetic diversity

\section{Introduction}

Une des particularités de l'espèce humaine est l'existence d'organisations sociales extrêmement variées et complexes. Les ethnologues distinguent trois composantes majeures de l'organisation sociale : la règle de filiation (qui affilie les individus à des groupes de parenté), la règle d'alliance (qui définit les alliances maritales possibles) et la règle de résidence (qui indique le lieu d'installation des nouveaux 
époux). Ces composantes varient d'une population à l'autre. D'après le Standard Cross-Cultural Sample (SCCS) [1], la filiation est cognatique (aussi dite bilatérale ou indifférenciée) dans $37 \%$ des populations, ce qui signifie que les enfants appartiennent à un réseau de parenté formé par leur famille maternelle et par leur famille paternelle. Mais dans la majorité des cas, la filiation est unilinéaire : les enfants appartiennent soit au groupe de filiation de leur père ( $40 \%$ des populations, filiation patrilinéaire), soit à celui de leur mère (14\% des populations, filiation matrilinéaire). Ces populations sont alors organisées en groupes de filiation, qu'il s'agisse de lignages, de clans ou de tribus (il existe d'autres modes de filiation plus rares que nous n'évoquons pas ici).

La règle d'alliance encourage le mariage entre certains types de cousins au premier degré dans environ $31 \%$ des populations, alors qu'elle les interdit dans $52 \%$ des populations. Le degré de polygamie varie également d'une population à l'autre, avec $17 \%$ de populations monogames, $52 \%$ de populations faiblement polygynes (moins de $20 \%$ des hommes ont plusieurs épouses), $31 \%$ de populations présentant une polygynie généralisée, et seulement $1 \%$ de populations polyandres.

La résidence post-maritale est patrilocale (chez le père du mari) ou virilocale (chez le mari, qui vit à proximité de son père) dans $64 \%$ des populations, matrilocale (chez la mère de la mariée) ou uxorilocale (chez la mariée, qui vit à proximité de sa mère) dans $20 \%$ des cas, ambilocale dans $6 \%$ des cas (à proximité de la famille de la mariée ou du marié), néolocale dans $5 \%$ des cas (lieu de résidence éloigné de celui des familles des deux époux) et avunculocale dans $4 \%$ des cas (à proximité de l'oncle maternel du marié).

\section{Prémices et promesses d'une discipline}

Par ces règles de filiation, de résidence et d'alliance, l'organisation sociale influence certains comportements matrimoniaux et démographiques clés pour l'évolution de la diversité génétique, notamment le niveau de structuration des populations, la migration des individus et la variance du nombre d'enfants qu'ils mettent au monde. De ce fait, les généticiens s'y intéressent de façon croissante, notamment afin de mieux décrire et retracer les dynamiques actuelles et passées de ces organisations sociales à partir des données génétiques. Ce domaine est prometteur dans la mesure où des données d'ADN ancien permettent de plus en plus d'analyser la diversité génétique des populations du passé et ainsi de tenter de retracer leurs organisations sociales (par exemple, [2-5]). Cependant, un préalable à cette inférence est l'étude des populations actuelles, et la description de la gamme de signatures génétiques laissées par un même type d'organisation sociale.
Ce champ de recherche s'appuie en grande partie, depuis une vingtaine d'années, sur l'ADN mitochondrial et le chromosome $\mathrm{Y}$, marqueurs génétiques transmis de façon uniparentale (par la mère pour l'ADN mitochondrial, par le père pour le chromosome $\mathrm{Y}$ ), et donc particulièrement adaptés pour étudier l'organisation sociale, car celle-ci induit souvent des comportements différents pour les hommes et les femmes [6]. En outre, les résultats à l'échelle mondiale montrent des différences de profils de diversité génétique pour le chromosome $\mathrm{Y}$ et l'ADN mitochondrial. D'une part, la diversité génétique se répartit de façon différente pour ces deux systèmes génétiques : une diversité intra-populationnelle moindre pour le chromosome Y que pour l'ADN mitochondrial et une diversité inter-populationnelle plus grande pour le chromosome Y que pour l'ADN mitochondrial [7]. D'autre part, la comparaison des arbres génétiques du chromosome $\mathrm{Y}$ et de l'ADN mitochondrial a fourni un des résultats les plus surprenants de la décennie passée dans le domaine de la génétique des populations humaines : une signature de réduction de la diversité génétique du chromosome $\mathrm{Y}$ (mais non de l'ADN mitochondrial) au Néolithique [8]. Ces profils de diversité différents pour le chromosome Y et l'ADN mitochondrial à l'échelle mondiale pourraient être le résultat de processus liés à l'organisation sociale, mais la nature exacte de ces processus reste débattue $[8,9]$.

Afin de mieux comprendre l'action des processus socioculturels sur les profils de diversité génétique uniparentaux, plusieurs équipes ont mené des études à une échelle locale, échelle à laquelle l'organisation sociale est connue et peut donc être mise en relation avec les signatures de diversité génétique. Une étude pionnière a ainsi montré en 2001 que la règle de résidence façonne les diversités mitochondriales et du chromosome $\mathrm{Y}$ de façon symétrique, avec une réduction de la diversité mitochondriale dans les populations matrilocales, et une réduction de la diversité du chromosome $\mathrm{Y}$ dans les populations patrilocales, comme attendu compte tenu des taux de migrations sexe-spécifiques induits par ces organisations sociales [10]. Cependant, la littérature plus récente a montré que ces attentes théoriques ne sont pas retrouvées dans d'autres populations [11-14]. Notamment, dans une étude à Sumatra, une population matrilocale montrait bien une réduction significative de la diversité génétique mitochondriale, mais aucune diminution significative de la diversité pour le chromosome $\mathrm{Y}$ n'a été mise en évidence dans le cas d'une population patrilocale voisine [14].

En outre, d'autres travaux ont mis en évidence que la règle de filiation module également la diversité génétique sexe-spécifique, avec une réduction de diversité génétique $\mathrm{du}$ chromosome $\mathrm{Y}$ et de la taille efficace des hommes (c'est-à-dire le nombre apparent d'hommes ayant une contribution génétique) dans les populations patrilinéaires [15-17] en comparaison à des populations cognatiques. Dans les systèmes polygynes, l'augmentation de la variance du succès 
reproducteur des hommes peut également mener à une réduction drastique de la diversité génétique du chromosome Y [18]. Ces travaux soulignent l'importance de considérer l'organisation sociale dans sa globalité pour comprendre les signatures qu'elle laisse au niveau génétique.

\section{Choix d'un terrain en Asie du Sud-Est et enjeux du projet}

Dans ce contexte, nous avons proposé de revisiter l'influence de l'organisation sociale sur la diversité génétique dans le cadre d'un projet financé par l'Agence nationale de la recherche. L'enjeu de ce projet était triple : tout d'abord, prendre en compte les différentes composantes de l'organisation sociale simultanément afin de distinguer l'influence exercée par chacune d'entre elles sur la diversité génétique. Notamment, dans le but de départager l'influence des processus de filiation et de résidence sur les profils de diversité sexe-spécifiques, nous avons inclus dans le jeu de données des populations ayant une même règle de résidence mais présentant des règles de filiation différentes. Le second enjeu était d'adopter une approche quantitative, non seulement pour la diversité génétique mais aussi pour les comportements de résidence, de filiation et d'alliance des individus via un questionnaire ethno-démographique détaillé. En effet, si certaines études ne retrouvent pas la signature attendue de l'organisation sociale sur la diversité génétique [11-14], cela peut être lié au fait que les individus ne suivent pas forcément la règle de résidence ou la règle de filiation [19]. Autrement dit, ce qui influence la diversité génétique, c'est le comportement réel des individus, plutôt que la règle qu'ils sont supposés suivre, et il était donc nécessaire de quantifier les processus de résidence, de filiation et d'alliance via un questionnaire détaillé, afin de mettre en relation ces données ethno-démographiques quantitatives avec la diversité génétique. Le troisième enjeu était d'étudier l'influence de l'organisation sociale sur la diversité génétique uniparentale mais aussi autosomale.

Nous avons donc sélectionné 12 populations d'Asie du Sud-Est (Cambodge et Laos), variables du point de vue de leur règles de filiation et de résidence : 4 populations à filiation matrilinéaire et résidence matrilocale (les Tampuan, Jarai, Kacho', et Prai), 4 populations à filiation patrilinéaire et résidence patrilocale (les Khmu', Ramet, Ta-oih, and Pacoh), et 4 populations à filiation cognatique et résidence matrilocale ou multilocale avec installation définitive préférentiellement dans le village natal de l'épouse (les Bunong, Khmer, Brao et Kreung). Ces populations sont toutes de langue austro-asiatique à l'exception des Jarai qui parlent une langue austronésienne. Ces populations partagent des environnements et des modes de subsistance proches (riziculture sur essarts, à l'exception des Khmers qui font de la rizicul- ture inondée). Nous avons ainsi limité l'effet de facteurs autres que l'organisation sociale pouvant influencer la diversité génétique, comme l'histoire du peuplement ou le mode de subsistance. Nous avons en outre privilégié des populations ayant fait l'objet au préalable d'études ethnographiques décrivant leur organisation sociale (les références sont données dans [20]).

Nous avons ainsi ouvert un nouveau terrain d'étude interdisciplinaire, alliant génétique des populations, linguistique, anthropologie et démographie. Nous avons ainsi échantillonné des données dans 61 villages du Cambodge et du Laos et interviewé 532 individus, ainsi que leur époux/épouse quand c'était possible. Nous avons récolté des informations ethno-démographiques pour chacun d'eux, ainsi que pour leurs parents, grands-parents, frères et sœurs, et enfants ; ce qui nous a permis de construire un jeu de données pour 3530 couples. Nous avons également collecté des données génétiques (obtenues à partir de prélèvements de salive) pour 1618 individus et produit des données génétiques haute densité pour 801 individus ( 1 à 2,5 millions de marqueurs génétiques). En parallèle, nous avons séquencé la région HV1 de l'ADN mitochondrial pour 619 individus et génotypé 17 microsatellites du chromosome Y pour 583 individus.

\section{Résidence et diversité génétique : de la règle à la pratique}

Nous avons tout d'abord calculé différents estimateurs de la diversité génétique uniparentale. Comme attendu, les populations matrilocales ont une diversité mitochondriale diminuée par rapport à celle des populations patrilocales, les populations multilocales étant intermédiaires. En revanche, nous n'avons pas observé de différences entre systèmes de parenté en termes de diversité du chromosome Y [20]. Ce résultat fait écho à de précédentes études qui n'ont pas retrouvé la signature attendue du mode de résidence sur la diversité génétique uniparentale [11-14]. Afin de comprendre ces observations, nous avons analysé les données ethnodémographiques dans le but de quantifier les flux migratoires d'hommes et de femmes dans ces populations.

Comme attendu, nous avons observé des flux migratoires de femmes réduits dans les populations matrilocales en comparaison aux populations patrilocales, les populations multilocales étant intermédiaires. En revanche, nous n'avons pas observé des flux migratoires d'hommes plus faibles dans les populations patrilocales que dans les populations matrilocales ou multilocales. Ce résultat peut sembler surprenant car nous avons par ailleurs observé que les règles matrilocales et patrilocales sont globalement respectées dans ces populations, et on aurait pu s'attendre à des flux migratoires restreints d'hommes au sein des populations patrilocales par rapport aux populations matrilocales. Pourquoi n'est-ce pas 
le cas ? Nos résultats montrent que les populations patrilocales, tout en respectant globalement leur règle de résidence patrilocale, sont assez flexibles quant à cette règle : elles tolèrent une proportion de couples matrilocaux plus élevée que la proportion de couples patrilocaux tolérée dans les populations matrilocales. Elles tolèrent également une proportion assez élevée de couples néolocaux. Ces proportions de couples matrilocaux et néolocaux contribuent à augmenter les migrations d'hommes, les rendant quasi identiques aux migrations d'hommes dans les populations matrilocales.

Nous pensons que cette égalité des flux migratoires d'hommes est une des explications possibles de l'égalité des niveaux de diversité du chromosome $\mathrm{Y}$ entre populations ayant des systèmes de parenté différents. Cela montre qu'il est crucial, pour réconcilier diversité génétique et système de parenté, de ne pas s'en tenir aux catégories fondées sur les règles, mais de quantifier les comportements de résidence : une population patrilocale peut être suffisamment flexible quant à cette règle pour que la signature de la patrilocalité ne soit pas retrouvée dans les données génétiques [20]. Cette explication nous parait plus parcimonieuse que l'explication alternative d'une transition récente dans l'organisation sociale de ces populations [14]. Ces résultats apportent de plus un éclairage nouveau aux inférences faites à partir des données d'ADN ancien. En particulier, nous montrons qu'il est hasardeux de passer d'un profil de diversité génétique à une règle de résidence comme cela est souvent fait [3]. En particulier, un haut niveau de diversité mitochondriale, comme rapporté dans cette étude, est compatible avec une règle patrilocale mais également avec une règle matrilocale modérément observée. Les signatures génétiques révèlent les comportements de résidence plutôt que les règles ellesmêmes.

\section{Analyse des données autosomales : l'effet du « puzzle matrilinéaire » sur la consanguinité}

A partir des données autosomales, nous avons étudié les patterns de consanguinité dans les différentes populations à l'étude. Nous avions deux hypothèses : 1) d'une part, nous pouvions nous attendre à des coefficients de consanguinité plus élevés dans les populations unilinéaires (c'est-à-dire matrilinéaires et patrilinéaires) que dans les populations cognatiques. En effet, $41 \%$ des populations unilinéaires, bien que pratiquant généralement l'exogamie lignagère ou clanique, ont tendance à favoriser le mariage avec un(e) cou$\sin (\mathrm{e})$ de premier degré (par exemple dans un système patrilinéaire avec la cousine croisée matrilatérale car celle-ci n'appartient pas au même clan), alors que seules $20 \%$ des populations cognatiques ont une telle préférence ; 2) la seconde hypothèse que nous avons envisagée dérive du
« puzzle matrilinéaire ». Celui-ci a été originellement conceptualisé par l'anthropologue A. Richards [21] à partir de l'observation des modes de résidence dans des populations matrilinéaires bantoues. En effet, contrairement à ce que l'on pourrait penser, un système matrilinéaire n'est pas le simple symétrique d'un système patrilinéaire : dans la très grande majorité des populations humaines, ce sont les hommes qui ont le pouvoir socio-politique, y compris dans les systèmes matrilinéaires [21-23]. De ce fait, les populations matrilinéaires doivent concilier une transmission de la filiation par les femmes, et une transmission de l'autorité sociopolitique par les hommes (gestion des affaires politiques à l'échelle du village, position d'autorité vis-à-vis des membres du groupe de filiation, pouvoir de décision concernant par exemple les alliances matrimoniales, la gestion des terres et/ou les biens matériels, etc.). Quand un homme se marie, si sa femme réside dans un village différent du sien, cela complique le maintien de son autorité dans son village natal, car il est moins souvent présent pour l'exercer. De ce fait, dans les populations matrilinéaires et/ou matrilocales, il y a une préférence pour des mariages au sein du village natal, comme l'avait noté Murdock dès 1949 : « Lorsque la résidence est matrilocale, un homme en se mariant, s'installe rarement dans une nouvelle communauté. Il prend ses possessions du foyer de ses parents et se déplace, pour ainsi dire, de l'autre côté de la rue, dans la partie du village où sa femme et la famille de sa femme habitent (traduction G. Ly) » [24]. Cela lui permettrait de rester à proximité des membres de son lignage ou clan (notamment ses sœurs et ses neveux et nièces) et de maintenir sa position d'autorité [25]. Si cette préférence pour des mariages à l'intérieur du village est avérée, cela pourrait augmenter les niveaux de consanguinité des populations matrilinéaires.

Nous avons estimé les coefficients de consanguinité individuels à partir des données génétiques de l'ensemble du génome à l'aide de la méthode Festim [26]. Nous n'avons pas observé de différences significatives entre les populations unilinéaires et cognatiques, contrairement à ce qui était attendu sous l'hypothèse (1). En revanche, nous avons mis en évidence des coefficients de consanguinité plus élevés dans les populations matrilocales que dans les populations patrilocales, comme attendu sous l'hypothèse (2) du « puzzle matrilinéaire » [27]. Nous avons par ailleurs montré grâce aux données ethno-démographiques, récoltées pour 3530 couples, que les populations matrilocales pratiquent plus fréquemment des mariages au sein du même village et que ce taux de mariages endogames est prédicteur du niveau de consanguinité [27]. Ces résultats soutiennent l'hypothèse que le « puzzle matrilinéaire » contribue à façonner les profils de consanguinité dans ces populations d'Asie du Sud-Est.

Nous avons par ailleurs montré que les niveaux de consanguinité et d'endogamie de village sont comparables dans les populations matrilocales, qu'elles soient matrilinéaires ou 
cognatiques, ce qui suggère que la règle de filiation n'est pas la source principale des contraintes exercées sur les migrations des hommes. Comme l'a expliqué A. Richards, dès lors que la règle de résidence est matrilocale, les hommes en se mariant avec une femme d'un autre village risquent de se retrouver en position de pouvoir moins favorable dans le village de leur femme (et de perdre la position d'autorité qu'ils avaient acquise dans leur village natal), une situation qui peut être évitée en se mariant avec une femme du même village.

Cette étude montre donc que les systèmes matrilocaux conduisent, sous un régime de domination masculine, à une plus grande endogamie et consanguinité génétique. Il reste à explorer si ce résultat est généralisable à d'autres populations matrilocales issues d'autres sphères géographicoculturelles. Il est probable que ce soit le cas dans la mesure où les anthropologues ont montré que l'endogamie est un trait partagé par les populations matrilocales.

\section{Conclusion}

Les résultats de ce projet montrent que les structures sociales laissent des signatures génétiques spécifiques. Ces signatures ouvrent de nouvelles perspectives, notamment mieux connaître la dynamique des structures sociales dans les populations humaines actuelles et passées. Cependant, ils appellent également à une certaine prudence lorsque les profils de diversité génétique sont utilisés pour inférer les règles sociales des populations à l'étude. Notamment, on ne peut pas déduire sans précaution une règle de résidence à partir d'un profil de diversité génétique issu de données archéogénétiques. Par exemple, un haut niveau de diversité pour le chromosome $\mathrm{Y}$ est compatible avec une règle matrilocale mais également avec une règle patrilocale modérément suivie. Nos résultats concernant la règle de filiation ne sont pas encore publiés, mais des travaux réalisés dans d'autres contextes socio-culturels ont montré que la règle de filiation peut également être contournée. Dans ce cas, les membres d'un même groupe de filiation ne sont pas plus génétiquement apparentés que les individus appartenant à des groupes de filiation différents [28]. Ces études montrent donc que les signatures génétiques révèlent les comportements de résidence et de filiation plutôt que les règles elles-mêmes et appellent donc à une certaine prudence dans l'interprétation faite à partir des profils de diversité génétique des populations anciennes.

Par ailleurs, notre étude montre que le « puzzle matrilinéaire » façonne les niveaux de consanguinité des populations humaines, identifiant ainsi un nouveau processus lié au système de parenté et à la domination masculine contribuant à moduler la consanguinité des populations humaines. Ces résultats ajoutent donc un niveau de complexité supplémen- taire aux interactions connues entre les facteurs socioculturels et les profils de diversité génétique.

Plus généralement, ces travaux enrichissent la théorie dite de la construction de niche [29] : les populations humaines construisent le théâtre écologique dans lequel elles évoluent, et en retour, ce théâtre exerce des pressions évolutives sur les populations humaines. Ce théâtre est souvent conçu comme matériel : les populations humaines innovent, fabriquent, cultivent, soignent. Mais certains traits immatériels comptent également : ainsi, l'organisation sociale, en définissant nos partenaires de jeu dans le théâtre de l'évolution, façonne, génération après génération, la diversité génétique des populations humaines.

Remerciements Cette étude a été financée par l'ANR (JC09_441218, projet SoGen). Nous remercions tous les volontaires qui ont participé à cette étude, Yves Buisson de l'Institut de la francophonie pour la médecine tropicale (IFMT) au Laos pour l'aide qu'il nous a apportée afin de faciliter le travail de terrain, ainsi que Evelyne Heyer et Michael Houseman pour les discussions éclairantes.

Liens d'intérêts : les auteurs déclarent ne pas avoir de liens d'intérêts.

\section{Références}

1. Murdock GP, White DR (1969) Standard Cross-Cultural Sample. Ethnology 8:329-69

2. Sánchez-Quinto F, Malmström H, Fraser M, Girdland-Flink L, Svensson EM, Simões LG, George R, Hollfelder N, Burenhult G, Noble G, et al (2019) Megalithic tombs in western and northern Neolithic Europe were linked to a kindred society. Proc Natl Acad Sci USA 116:9469-74

3. Knipper C, Mittnik A, Massy K, Kociumaka C, Kucukkalipci I, Maus M, Wittenborn F, Metz SE, Staskiewicz A, Krause J, et al (2017) Female exogamy and gene pool diversification at the transition from the Final Neolithic to the Early Bronze Age in central Europe. Proc Natl Acad Sci USA 114:201706355

4. Kennett DJ, Plog S, George RJ, Culleton BJ, Watson AS, Skoglund P, Rohland N, Mallick S, Stewardson K, Kistler L, et al (2017) Archaeogenomic evidence reveals prehistoric matrilineal dynasty. Nat Commun 8:1-9

5. Mary L, Zvénigorosky V, Kovalev A, Gonzalez A, Fausser J.L, Jagorel F, Kilunovskaya M, Semenov V, Crubézy E, Ludes B, et al (2019) Genetic kinship and admixture in Iron Age ScythoSiberians. Hum Genet 138:411-23

6. Heyer E, Chaix R, Pavard S, Austerlitz F (2012) Sex-specific demographic behaviours that shape human genomic variation. Mol Ecol 21:597-612

7. Lippold S, Xu H, Ko A, Li M, Renaud G, Butthof A, Schröder R, Stoneking M (2014) Human paternal and maternal demographic histories: insights from high-resolution $\mathrm{Y}$ chromosome and mtDNA sequences. Investig Genet 5:13

8. Karmin M, Saag L, Vicente M, Wilson Sayres MA, Järve M, Gerst Talas U (2015) A recent bottleneck of Y chromosome diversity coincides with a global change in culture. Genome Res 25(4):459-66 
9. Zeng TC, Aw AJ, Feldman MW (2018) Cultural hitchhiking and competition between patrilineal kin groups explain the postNeolithic Y-chromosome bottleneck. Nat Commun 9:2077

10. Oota $\mathrm{H}$, Settheetham-Ishida W, Tiwawech D, Ishida T, Stoneking M (2001) Human mtDNA and Y-chromosome variation is correlated with matrilocal versus patrilocal residence. Nat Genet 29:20-1

11. Kumar V, Langstieh BT, Madhavi K. V, Naidu VM, Singh HP, Biswas S, Thangaraj K, Singh L, Reddy BM (2006) Global patterns in human mitochondrial DNA and Y-chromosome variation caused by spatial instability of the local cultural processes. PLoS Genet 2:e53

12. Besaggio D, Fuselli S, Srikummool M, Kampuansai J, Castrì L, Tyler-Smith C, Seielstad M, Kangwanpong D, Bertorelle G (2007) Genetic variation in Northern Thailand Hill Tribes: origins and relationships with social structure and linguistic differences. BMC Evol Biol 7(Suppl 2):S12

13. Verdu P, Becker NSA, Froment A, Georges M, Grugni V, Quintana-Murci L, Hombert JM, Van Der Veen L, Le Bomin S, Bahuchet S, et al (2013) Sociocultural behavior, sex-biased admixture, and effective population sizes in central African pygmies and non-pygmies. Mol Biol Evol 30:918-37

14. Gunnarsdóttir ED, Nandineni MR, Li M, Myles S, Gil D, Pakendorf B, Stoneking M (2011) Larger mitochondrial DNA than Y-chromosome differences between matrilocal and patrilocal groups from Sumatra. Nat Commun 2:228

15. Chaix R, Quintana-Murci L, Hegay T, Hammer MF, Mobasher Z, Austerlitz F, Heyer E (2007) From Social to Genetic Structures in Central Asia. Curr Biol 17(1):43-8

16. Ségurel L, Martínez-Cruz B, Quintana-Murci L, Balaresque $P$, Georges M, Hegay T, Aldashev A, Nasyrova F, Jobling MA, Heyer E, et al (2008) Sex-specific genetic structure and social organization in Central Asia: insights from a multi-locus study. PLoS Genet 4:e1000200

17. Marchi N, Hegay T, Mennecier P, Georges M, Laurent R, Whitten M, Endicott P, Aldashev A, Dorzhu C, Nasyrova F, et al (2017) Sex-specific genetic diversity is shaped by cultural factors in Inner Asian human populations. Am J Phys Anthropol 162(4): 1-14

18. Kayser M, Brauer, S, Weiss G, Schiefenhövel W, Underhill P, Shen P, Oefner P, Tommaseo-Ponzetta M, Stoneking M (2003)
Reduced Y-chromosome, but not mitochondrial DNA, diversity in human populations from West New Guinea. Am J Hum Genet 72:281-02

19. Sahlins M (1965) On the Ideology and Composition of Descent Groups. Man $65: 104-7$

20. Ly G, Alard B, Laurent R, Lafosse S, Toupance B, Monidarin C, Diffloth G, Bourdier F, Evrard O, Pavard S, et al (2018) Residence rule flexibility and descent groups dynamics shape uniparental genetic diversities in South East Asia. Am J Phys Anthropol 165:480-91

21. Richards AI (1950) Some Types of Family Structure Amongst the Central Bantu. In: Radcliffe-Brown A, Forde D (eds.) African Systems of Kinship and Marriage. Oxford University Press, Oxford, pp 207-51

22. Héritier F (1996) Masculin, Féminin. La pensée de la différence, Odile Jacob, Paris, $336 \mathrm{p}$

23. Mathieu NC, (2007) Introduction. In: Mathieu NC (ed.) Une maison sans fille est une maison morte. La personne et le genre en sociétés matrilinéaires et/ou uxorilocales. Maison des sciences de l'homme, Paris, $503 \mathrm{p}$

24. Murdock GP (1949) Social Structure, Macmillian Company, London, $387 \mathrm{p}$

25. Kloos P (1963) Matrilocal residence and local endogamy: Environmental knowledge or leadership. Am Anthropol 65:854-62

26. Leutenegger A-L, Prum B, Génin E, Verny C, Lemainque A, Clerget-Darpoux F, Thompson E (2003) Estimation of the inbreeding coefficient through use of genomic data. Am J Hum Genet 73:516-23

27. Ly G, Laurent R, Lafosse S, Monidarin C, Diffloth G, Bourdier F, Evrard O, Toupance B, Pavard,S, Chaix R (2019). From matrimonial practices to genetic diversity in Southeast Asian populations: The signature of the matrilineal puzzle. Philos Trans R Soc B Biol Sci 374(1780):20180434

28. Raaum RL, Al-Meeri A, Mulligan CJ (2013) Culture modifies expectations of kinship and sex-biased dispersal patterns: A case study of patrilineality and patrilocality in tribal yemen. Am J Phys Anthropol 150:526-38

29. Laland KN, Odling-Smee J, Myles S (2010) How culture shaped the human genome: bringing genetics and the human sciences together. Nat Rev Genet 11:137-48 